\title{
Avaliação do Ciclo de Vida na construção civil brasileira: análise de impactos ambientais
}

Uma vez que a construção civil é um dos setores da economia que mais emite gases de efeito estufa e consome energia em todo o mundo, a análise do ciclo de vida dos insumos de uma construção torna-se bastante importante atualmente. A Avaliação do Ciclo de Vida analisa os impactos ambientais produzidos nas diversas fases do processo construtivo, desde a extração e fabricação de matérias-primas até a renovação ou demolição da estrutura, colaborando na tomada de decisões e podendo levar a uma redução do impacto ambiental de construções. Estes impactos podem ser avaliados através das emissões atmosféricas, consumo de recursos naturais, demandas energéticas e geração de resíduos. Assim, este trabalho tem como objetivo utilizar a metodologia da Avaliação do Ciclo de Vida para calcular os impactos de demanda de energia e a emissão de CO2 da construção de uma escola. O projeto é chamado de 'Espaço Educativo Urbano' e é proposto pelo Fundo Nacional de Desenvolvimento da Educação. Para calcular os resultados, foram adotadas diversas premissas baseadas no cenário brasileiro e, analisando tais cenários, foi possível perceber que existem diversas maneiras de facilmente se alcançar uma redução de cerca de $20 \%$ nos impactos ambientais relacionados tanto com a demanda de energia quanto com a emissão de $\mathrm{CO} 2$. Além disso, os resultados mostraram que existe a possibilidade de reduzir o impacto futuro de outras construções através da reciclagem de materiais.

Palavras-chave: Construção civil; Sustentabilidade; Avaliação do Ciclo de Vida; Impacto Ambiental.

\section{Life Cycle assessment in Brazilian civil construction: analysis of environmental impacts}

\begin{abstract}
Since civil construction is one of the sectors of the economy that emits the most greenhouse gases and consumes energy around the world, the analysis of the life cycle of building inputs is currently very important. The Life Cycle Assessment analyzes the environmental impacts produced in the various stages of the construction process, from the extraction and manufacture of raw materials to the renovation or demolition of the structure, collaborating in decision making and can lead to a reduction in the environmental impact of buildings. These impacts can be assessed through atmospheric emissions, consumption of natural resources, energy demands and waste generation. Thus, this work aims to use the Life Cycle Assessment methodology to calculate the impacts of energy demand and CO2 emissions from the construction of a school. The project is called 'Urban Educational Space' and is proposed by the National Education Development Fund. To calculate the results, several assumptions were adopted based on the Brazilian scenario and, analyzing such scenarios, it was possible to see that there are several ways to easily achieve a reduction of about $20 \%$ in environmental impacts related to both energy demand and emission of CO2. Furthermore, the results showed that there is a possibility to reduce the future impact of other constructions by recycling materials.
\end{abstract}

Keywords: Construction; Sustainability; Life Cycle Assessment; Environmental impact.

Topic: Engenharia Civil

Reviewed anonymously in the process of blind peer
Received: $17 / \mathbf{1 2} / \mathbf{2 0 2 0}$

Approved: 10/03/2021
Deborah Attié (1)

Pontifícia Universidade Católica do Rio de Janeiro, Brasil http://orcid.org/0000-0003-3536-7083 deborahattie@gmail.com

Fernanda de Andrade Salgado (iD)

Pontifícia Universidade Católica do Rio de Janeiro, Brasil http://lattes.cnpq.br/2585591562683294

http://orcid.org/0000-0001-8900-5964

fernanda.salgado@puc-rio.br

\section{Referencing this:}

ATTIÉ, D.; SALGADO, F. A.. Avaliação do Ciclo de Vida na construção civil brasileira: análise de impactos ambientais. Engineering Sciences, v.9, n.1, p.18-27, 2021. DOI: http://doi.org/10.6008/CBPC23183055.2021.001.0003 


\section{INTRODUÇÃO}

Após a Conferência de Estocolmo em 1972, envolvendo 113 países, foram criadas agências que passaram a se ocupar da questão ambiental, como por exemplo a EPA (Environmental Protection Agency) dos Estados Unidos e a Sema (Secretaria Especial do Meio Ambiente) no Brasil. Além disso, em 1987, o conceito de desenvolvimento sustentável foi introduzido pela Comissão Brundtland no relatório "Nosso Futuro Comum" (IPIRANGA et al., 2011).

Especificamente com relação à construção civil, a geração do resíduo de construção e demolição (RCD) tem crescido progressivamente nos últimos anos. Segundo a ABRELPE (Associação Brasileira de Empresas de Limpeza Pública e Resíduos Especiais), em 2018 foram produzidos, aproximadamente, 44 milhões de toneladas de RCD no Brasil (122 mil toneladas por dia). Essa quantidade representa cerca de 56\% do total de resíduos sólidos urbanos gerados no país. Quando não gerenciados propriamente, tais resíduos podem causar um grande impacto ambiental através da poluição do solo, ar e água, e também nas atividades econômicas e sociais, em função da obstrução de vias públicas, comprometimento da qualidade do ambiente e da paisagem local e saúde das pessoas (PINTO, 1999).

Nesse contexto, a Avaliação do Ciclo de Vida (ACV) se destaca como uma ferramenta que permite avaliar o impacto ambiental potencial associado à um produto ou atividade durante a sua vida útil. A ACV permite analisar os estágios do ciclo de vida e fazer escolhas alternativas de produtos, processos ou serviços, a partir de um inventário de entradas e saídas do sistema considerado (SOARES et al., 2006; COLTRO et al., 2017). Assim, a Avaliação do Ciclo de Vida (ACV) de edificações tem sido gradualmente utilizada para identificar os melhores cenários para gestão de resíduos, que podem prevenir ou reduzir os impactos negativos nos ecossistemas e na saúde humana (BUTERA et al., 2015).

Após diversas conferências, o primeiro documento voltado à padronização da metodologia da ACV foi publicado em 1993 e chamado de "SETAC Guidelines for Life Cycle Assessment - a Code of Practice" e a partir desse documento, em 1997, foi gerada a série de normas ISO 14040 (COLTRO et al., 2007). A sugestão da norma ABNT NBR ISO 14040:2009 para um estudo de ACV inclui quatro fases: definição de objetivo e escopo, análise de inventário do ciclo de vida (ICV), avaliação de impactos de ciclo de vida (AICV) e interpretação de resultados.

De maneira resumida, na primeira etapa é definido o objetivo do estudo, as fronteiras do sistema e a unidade funcional. Já a fase de análise de inventário, envolve coletar os dados necessários para o estudo e avaliar as entradas e saídas relevantes para o sistema. Na fase seguinte, de avaliação de impacto de ciclo de vida, os dados gerados na análise de inventário são associados a impactos ambientais específicos, para que estes impactos possam ser avaliados. Finalmente, na etapa de interpretação dos resultados, as informações obtidas anteriormente são combinadas e interpretadas de acordo com os objetivos definidos no início do estudo (COLTRO et al., 2007).

Para a construção civil, as etapas do ciclo de vida de uma edificação são a Produção, a Construção, a Manutenção e a Demolição/Fim de Vida. Podem ser consideradas também as etapas de Reciclagem e Reuso, 
que são definidas como potenciais benefícios. A etapa de produção inclui a extração de insumos, o transporte e a manufatura. Nesse momento é definida a quantidade de material necessária para a realização da obra. Na etapa seguinte, de Construção, é analisada a metodologia construtiva e instalação do edifício, além do transporte dos materiais tanto entre a loja e o canteiro de obras quanto entre o canteiro de obras e o descarte dos resíduos da obra. A etapa de Manutenção engloba o uso, manutenção, reparos, reposições e reformas da edificação. Para a fase de Demolição/Fim de Vida é considerado o processamento dos resíduos, além do descarte ou destinação dos mesmos. Para as etapas de Reciclagem e Reuso, consideradas como benefícios, é definida a quantidade de material que poderá ser reutilizado ou reciclado (adaptado de BSI, 2011).

Assim, nessa pesquisa será apresentado o estudo de caso da construção de uma escola, buscando analisar os seus impactos ambientais através da metodologia de Avaliação do Ciclo de Vida. Foram analisados cinco diferentes cenários, baseados na realidade brasileira e de outros países, como EUA e países da União Europeia. Para tal comparação, foram considerados indicadores de reciclagem, descarte, vida útil e perda de material. Assim, buscou-se identificar as categorias de impacto com contribuições mais significativas e definir possíveis estratégias para minimizar os efeitos causados ao meio ambiente.

\section{RELATO}

O objeto desse estudo de caso é um projeto de escola chamado "Espaço Educativo Urbano", cuja proposta pode ser encontrada na página do Fundo Nacional de Desenvolvimento da Educação. A edificação possui 12 salas, todas em um único pavimento térreo, com capacidade de até 780 alunos divididos em dois turnos (matutino e vespertino), além de mais 390 alunos em período integral. O projeto do FNDE considera como ideal a implantação da escola em terreno retangular com medidas de $80 \mathrm{~m}$ de largura por $100 \mathrm{~m}$ de profundidade, com declividade máxima de 3\%. Além disso, o conjunto deve ser formado por 8 blocos distintos, separados em administrativo, pedagógico e serviços, como cozinha. A técnica construtiva deve ser simples, adotando materiais facilmente encontrados e sem necessidade de mão-de-obra especializada, para que facilite a construção do edifício em qualquer região do Brasil.

Para o estudo de caso em questão, a escola foi considerada locada no endereço Rua Marquês de São Vicente, entre os números 96 e 124 - Gávea, Rio de Janeiro, local onde há atualmente um terreno livre para a construção e instalação do canteiro de obras. Para a Avaliação do Ciclo de Vida, foi criado um cenário base (Cenário 1) apoiado em dados da construção civil brasileira.

Para a etapa de Produção, foi utilizada a planilha orçamentária disponibilizada pelo FNDE e relatórios com as referências em insumos e composições de serviço do Sistema Nacional de Pesquisa de Custos e Índices (SINAPI, 2018) e da Secretaria de Estado de Infraestrutura e Obras - SEINFRA (CEARÁ, 2019). Além disso, foi necessário estabelecer os fatores de demanda de energia e de emissão de $\mathrm{CO}_{2}$ incorporados em cada material para definir o impacto ambiental dos mesmos. Esses fatores foram obtidos da base de dados ICV da Ecoinvent (versão 3.4), uma associação sem fins lucrativos fundada por diversos institutos do ETH Domain (Domain of the Swiss Federal Institutes os Technology - união entre universidades suíças e instituições de 
pesquisa).

Para a etapa de Construção, foi determinado o percentual de perda de cada material, baseado nos estudos da FINEP, Pinto (1989) e Agopyan. Também foram estabelecidas as distâncias entre o canteiro de obras e a loja de materiais e o aterro sanitário. A loja foi definida a partir do bairro do canteiro de obras (terreno), levando em conta a distância entre os dois e seu tamanho, facilitando a compra de todos os materiais em apenas um lugar. Para o descarte de material foi escolhido o aterro sanitário CTR Seropédica (Centro de Tratamento de Resíduos), onde é descartado atualmente todo o resíduo de construção e demolição (RCD) do Rio de Janeiro. Nesta etapa foi ainda definido que a forma de transporte dos insumos e resíduos seria por caminhão. A definição da forma de transporte é importante pois, para cada tipo, há um coeficiente de impacto ambiental diferente. Para caminhões, os coeficientes considerados foram 3,23 MJ/tonelada.km e 0,19 $\mathrm{KgCO}_{2}$ eq/tonelada.km (BRIBIÁN et al., 2011).

Para a etapa de Manutenção, foi adotada a vida útil dos materiais de acordo com a norma ABNT NBR 15575:2013, considerando a vida útil da estrutura de 50 anos. Neste estágio, foi avaliada a possibilidade de aumentar a vida útil de esquadrias e PVC, com o intuito de analisar a variação no impacto ambiental. Foram analisados apenas esses dois materiais pois eles são de fácil acesso e não requerem muito esforço para realização de trocas, caso necessário. No caso de esquadrias, por exemplo, para aumentar sua vida útil e evitar trocas desnecessárias, é preciso avaliar o tratamento que o material recebe desde a chegada na obra até a instalação, padronizar o manuseio do produto, calcular corretamente os vãos onde as portas serão inseridas, entre outros (ABIMCI, 2018).

Na etapa de Demolição, e consequentemente de transporte dos resíduos, foram consideradas as distâncias entre o canteiro, aterro e centro de reciclagem, além dos coeficientes de impacto da frota utilizada - os mesmos valores considerados para a etapa de Construção (BRIBIÁN et al., 2011). O Centro de Reciclagem Rio (CRR) escolhido foi o de Guadalupe, pelo fato de ser a matriz do CRR.

Para o Fim de Vida, baseado nos dados da construção civil brasileira, foi considerado que apenas $20 \%$ dos resíduos vão para reciclagem e o restante (80\%) é direcionado para aterro sanitário. Já com relação aos benefícios, foi adotada a premissa de que 10\% do material da etapa "Produção" vem do reuso (ADDIS, 2010).

Além do Cenário 1 (cenário base), foram criados outros cinco cenários com o objetivo de analisar os possíveis efeitos da modificação das premissas adotadas inicialmente (Tabela 1).

Tabela 1: Descrição dos cenários analisados.

\begin{tabular}{|l|l|l|l|l|l|l|}
\hline Cenário & $\mathbf{1}$ (base) & $\mathbf{2}$ & $\mathbf{3}$ & $\mathbf{4}$ & $\mathbf{5}$ & $\mathbf{6}$ \\
\hline Reciclagem (\%) & $20 \%$ & $20 \%$ & $40 \%$ & $40 \%$ & $60 \%$ & $40 \%$ \\
\hline Descarte (\%) & $80 \%$ & $80 \%$ & $60 \%$ & $60 \%$ & $40 \%$ & $60 \%$ \\
\hline Distância descarte (km) & 80 & 80 & 80 & 80 & 80 & 80 \\
\hline Distância reciclagem (km) & 35,3 & 35,3 & 35,3 & 35,3 & 35,3 & 35,3 \\
\hline Local de aquisição (km) & 17 & 17 & 17 & 17 & 17 & 17 \\
\hline Vida útil esquadrias (anos) & 10 & 10 & 10 & 20 & 20 & 20 \\
\hline Vida útil PVC (anos) & 20 & 20 & 20 & 30 & 30 & 30 \\
\hline Perda na construção (\%) & $20-30 \%$ & $20-30 \%$ & $20-30 \%$ & $20-30 \%$ & $10 \%$ & $15 \%$ \\
\hline Coeficientes de impacto no “Fim de Vida"? & Não & Sim & Não & Não & Não & Não \\
\hline Material de “Produção" vindo do reuso (\%) & $10 \%$ & $10 \%$ & $10 \%$ & $20 \%$ & $20 \%$ & $20 \%$ \\
\hline Modal de transporte utilizado & Caminhão & Caminhão & Caminhão & Caminhão & Caminhão & Caminhão \\
\hline
\end{tabular}


Como pode ser observado na Tabela 1, o segundo cenário é análogo ao cenário base (Cenário 1), com exceção da etapa "Fim de Vida", pois considera coeficientes de impactos ambientais gerados pelo descarte e pela reciclagem (NG et al., 2015; POLITECNICO DI MILANO, 2018). O objetivo da avaliação deste cenário é compreender o impacto causado pelo aumento da reciclagem e, consequentemente, redução do descarte. É importante destacar que esses coeficientes refletem apenas os principais materiais de uma obra, como o concreto e o alumínio. Assim, por falta de coeficientes de diversos materiais para reciclagem ou para seu descarte (aterro sanitário), não foram considerados os impactos provocados por essas atividades na etapa "Fim de Vida" em nenhum dos outros cenários, descritos a seguir. Portanto, na etapa de "Fim de Vida" dos cenários 1 e 3 ao 6, apenas foram calculados os impactos poupados no futuro devido à reciclagem dos resíduos (benefícios).

Ainda na Tabela 1, é possível perceber que a diferença entre o Cenário Base e o Cenário 3 se dá no percentual de material que vai para a reciclagem (de 20\% para 40\%) (US EPA, 2003). Já no Cenário 4, foram feitas alterações na vida útil de esquadrias e PVC de 10 e 20 anos para 20 e 30 anos ( $\mathrm{ABIMCl}, 2018)$, respectivamente, além do aumento do percentual de material utilizado que vem de reuso de 10\% para $20 \%$ (ADDIS, 2010). Para o Cenário 5 foram mantidas as premissas do Cenário 4, com exceção da taxa de reciclagem e do percentual de perda na construção. Neste cenário foi utilizada uma taxa de reciclagem de $60 \%$, compatível com países como a Holanda, Dinamarca e Alemanha, que reciclam entre $50 \%$ e $90 \%$ do total do RCD gerado (ANGULO, 2005); já a taxa de perda de 10\% foi baseada em dados da Holanda (BOSSINK et al., 1996). Finalmente, o Cenário 6 foi baseado no Cenário 4, mas com uma taxa de perda na construção de 15\%, um valor intermediário entre a realidade brasileira (20-30\%) e a realidade da Holanda (10\%) (BOSSINK et al., 1996), com o objetivo de criar um cenário mais realístico para o estudo de caso em questão.

\section{DISCUSSÃO}

Com base nas premissas mencionadas anteriormente, obteve-se para Cenário 1 (cenário base) os resultados indicados na Figura 1 para demanda de energia e emissões de $\mathrm{CO}_{2}$ incorporadas no total. É possível perceber que o benefício potencial, ou seja, o impacto futuro poupado para este cenário base é extremamente pequeno (menor do que $1 \%$ ). Isso se deve ao fato de que foi considerado, na etapa de fim de vida, que apenas $20 \%$ dos resíduos seriam reciclados. No caso dos países europeus, este percentual está mais próximo de 50\%, taxa onde o benefício obtido passa a ser relevante, em torno de 10\% (ANGULO, 2005).

Conforme comentado, a etapa de fim de vida levando em conta os coeficientes de impacto do descarte e reciclagem dos materiais foi considerada apenas no Cenário 2 (Figura 2). Como esperado, quando esses coeficientes são considerados, há um aumento do impacto ambiental de todo o processo. A principal diferença deste cenário é que ele retrata com mais transparência a realidade do ciclo de vida da escola.

Para que seja possível comparar diferentes modelos e tamanhos de escolas, foi calculada a demanda de energia e a emissão de $\mathrm{CO}_{2}$ incorporadas por aluno e por $\mathrm{m}^{2}$ (Figura 3). Foram considerados 780 alunos e $3.228 \mathrm{~m}^{2}$, seguindo a proposta do projeto original do FNDE. É importante ressaltar que a unidade de referência "aluno" é de maior interesse para pedagogos e para o Governo, pois quanto mais estudantes 


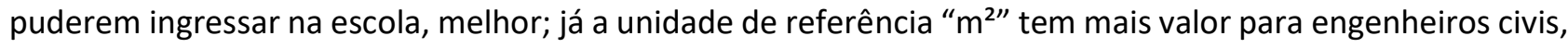
arquitetos e construtores, por ser uma das unidades de referência normalmente utilizada em negociações de construção (QUEIROZ, 2001).

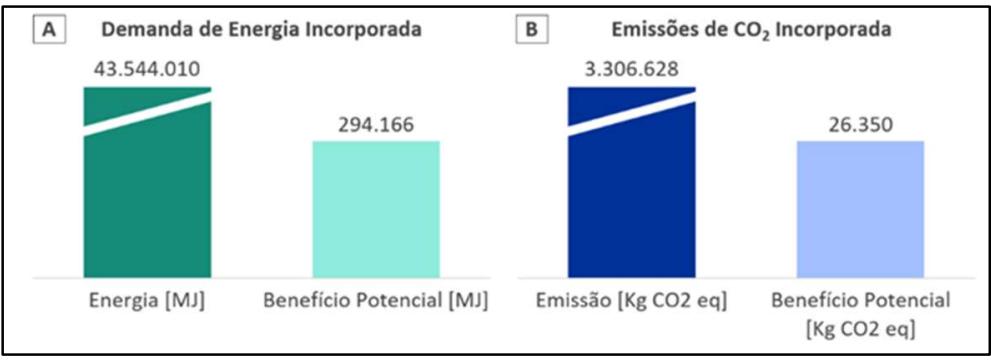

Figura 1: Demanda de energia incorporada e emissão de $\mathrm{CO}_{2}$ incorporada totais - cenário base.

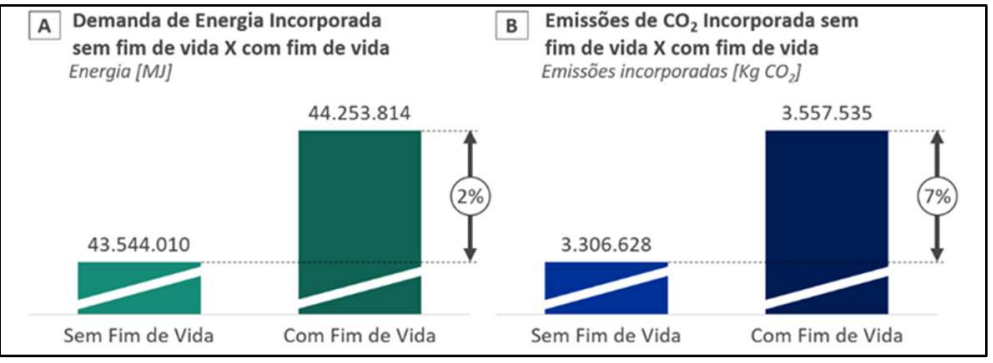

Figura 2: Impactos sem fim de vida versus com fim de vida (com coeficientes).

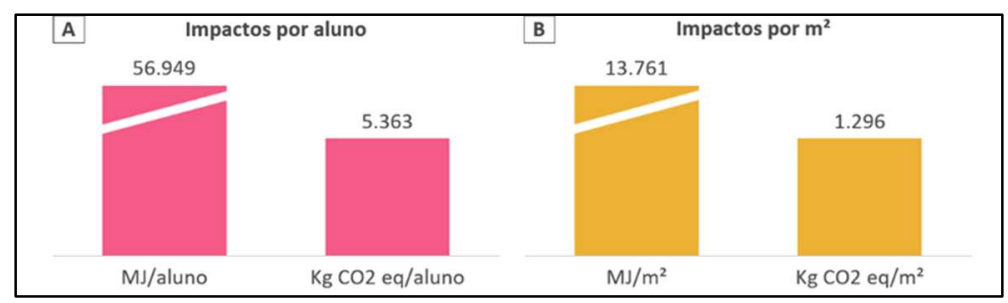

Figura 3: Impactos por aluno e por $\mathrm{m}^{2}$.

A Figura 4 mostra a demanda de energia e a emissão de $\mathrm{CO}_{2}$ incorporadas durante cada etapa da construção considerando o Cenário 1 (cenário base). É possível perceber que as etapas de “Produção" e "Manutenção" são aquelas que mais promovem um impacto ambiental, sendo responsáveis por 68\% e 31\% da demanda de energia incorporada e $74 \%$ e $24 \%$ da emissão de $\mathrm{CO}_{2}$ incorporada, respectivamente. Para ambas as análises, quando essas duas etapas são somadas, há uma contribuição quase total. Esse resultado explicita a importância de priorizar tais etapas construtivas para que seja possível obter uma redução do impacto ambiental da escola.

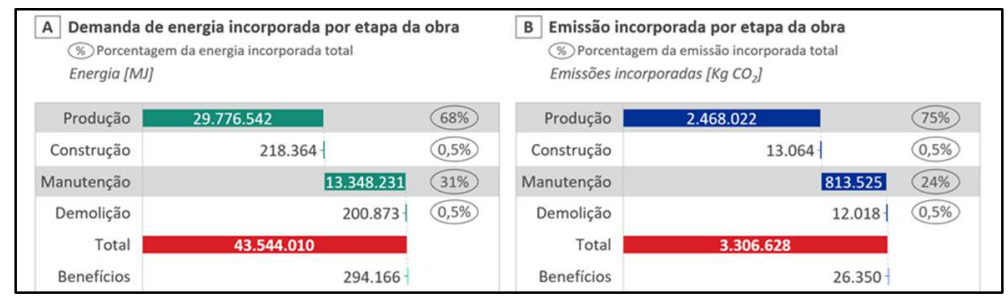

Figura 4: Energia e emissão de $\mathrm{CO}_{2}$ incorporada por etapa da obra.

As Figuras 5a e 5b mostram a demanda de energia e a emissão de $\mathrm{CO}_{2}$ de cada tipo de serviço da etapa "Produção". Nesta etapa, a execução do sistema de vedação vertical interno e externo (paredes) é o 
serviço que mais contribui para a demanda de energia incorporada ( $35 \%)$ e, por isso, deve ser o foco para alternativas sustentáveis. Em relação a emissão de $\mathrm{CO}_{2}$, o serviço com maior impacto durante a etapa de "Produção" também é o sistema de vedação (paredes) ( 73\%). Já na etapa de "Manutenção", as esquadrias têm uma forte influência na demanda de energia incorporada, com cerca de 76\%, e também na emissão de $\mathrm{CO}_{2}$, com aproximadamente $74 \%$ (Figuras $5 \mathrm{c}$ e $5 \mathrm{~d}$ ).

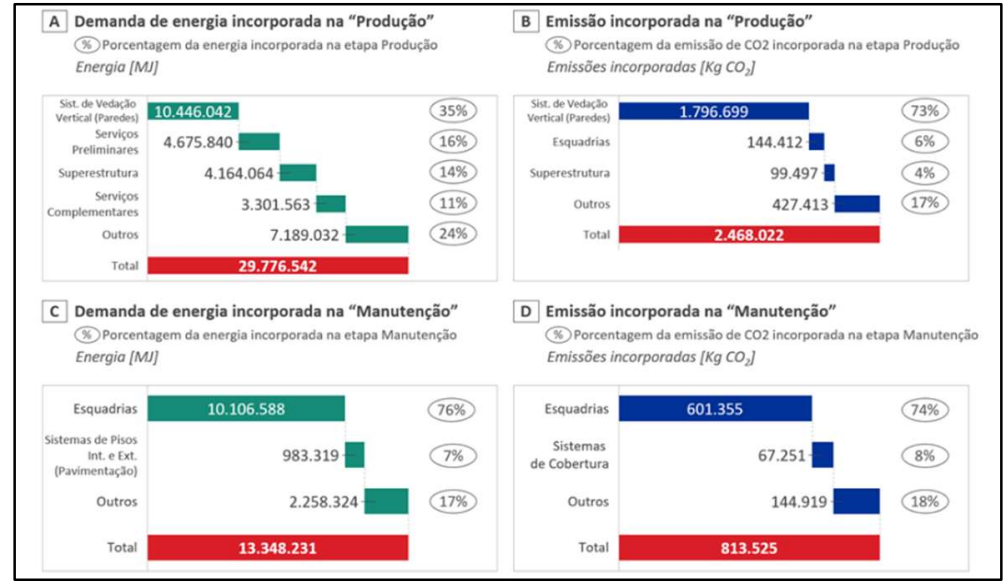

Figura 5: Energia e emissão de $\mathrm{CO}_{2}$ incorporadas na Produção e Manutenção.

Para reduzir o impacto ambiental das diferentes etapas de construção de uma escola, existem diversas soluções a serem exploradas. Em relação aos insumos, uma iniciativa seria substituir materiais comuns por reciclados, gerando um impacto ambiental mais baixo, sem que houvesse uma variação grande no custo da obra. Por exemplo, substituir o concreto armado convencional por outros tipos de concreto, como concreto celular autoclavado (CCA), concreto "vazado" (hollow concrete), stabilized soil block, pode proporcionar uma redução de $30 \%$ a $40 \%$ da demanda de energia incorporada para toda a edificação (HUBERMAN et al., 2008). Outra iniciativa muito relevante para a redução do impacto ambiental seria alterar o processo construtivo. Por exemplo, o concreto pré-fabricado emite $20 \%$ menos $\mathrm{CO}_{2}$ e demanda $43 \%$ menos energia por metro quadrado de construção do que concreto produzido in loco (COLE, 2000).

Em relação ao transporte, existem diversas iniciativas que resultariam em um menor impacto no meio ambiente, como: a) consolidar os fornecedores não só para garantir ganho de escala, mas também reduzir o impacto do combustível; b) selecionar fornecedores mais próximos ou ainda aqueles que utilizem modais distintos e tenham um processo de produção mais eficientes. O caminhão, por exemplo, possui um impacto ambiental aproximadamente quatro vezes superior ao trilho de carga (BRIBIÁN et al., 2011). Porém, no contexto da construção civil, esse meio de transporte se adequa pouco às situações de uma obra uma vez que normalmente as edificações estão locadas em uma parte mais urbana e as lojas de materiais, aterros e centros de reciclagem não se localizam em regiões onde há ferrovias. Além disso, no Brasil não há ferrovias suficientes para que esse meio de transporte seja eficaz na movimentação de materiais e resíduos.

Reduzir perdas de materiais também geraria um resultado muito positivo. No Brasil, em torno de $25 \%$ de todo o material adquirido não é bem utilizado e acaba sendo desperdiçado. Enquanto isso, outros países, como a Holanda, possuem uma média de desperdício de 9\% (BOSSINK et al., 1996). Essa grande diferença percentual mostra que no Brasil há muita oportunidade de melhoria na gestão dos materiais. Outra 
importante iniciativa seria reutilizar ou reciclar esse material desperdiçado ao longo do processo construtivo. Estima-se que $80 \%$ do material desperdiçado pode ser reutilizado ou reciclado ( $\mathrm{ABIMCl}, 2018)$. Na prática, algumas ações possíveis seriam, por exemplo, a reutilização de fôrmas e designação de um responsável por treinar os operários e coordenar a gestão dos resíduos.

Na etapa de "Manutenção", adotar medidas que aumentem a vida útil dos materiais também reduziria o impacto ambiental. Por exemplo, é possível utilizar esquadrias de madeira que possuem 20 anos de vida útil ao invés dos 13 anos previstos para as esquadrias de alumínio ( $\mathrm{ABIMCl}, 2018)$. Para isso, investir na qualidade do material e no cuidado ao transportá-lo e manuseá-lo é de extrema importância. Além disso, é importante que o edifício tenha uma ventilação adequada para evitar que a umidade do ar gere a necessidade de manutenção da esquadria.

Para as etapas de "Demolição" e "Fim de Vida", ações como alterar o processo de disposição dos materiais (reciclagem, aterro e incineração, por exemplo) e selecionar locais mais próximos para realizar a disposição podem trazer uma redução no impacto ambiental. Além disso, é importante definir um plano de demolição responsável que identifique as quantidades e tipos de materiais contaminantes e os materiais que podem ser reutilizados ou reciclados. Proteger o solo e a vegetação durante a demolição e agendar a retirada dos materiais que podem ser reutilizados e reciclados antes da demolição são iniciativas importantes para reduzir a demanda de energia e emissões de $\mathrm{CO}_{2}$ incorporadas nestas etapas. A Figura 6 mostra os resultados de todos os cenários, com exceção do Cenário 2 que não é comparável aos demais uma vez que considera os coeficientes de impacto do descarte e reciclagem dos materiais.

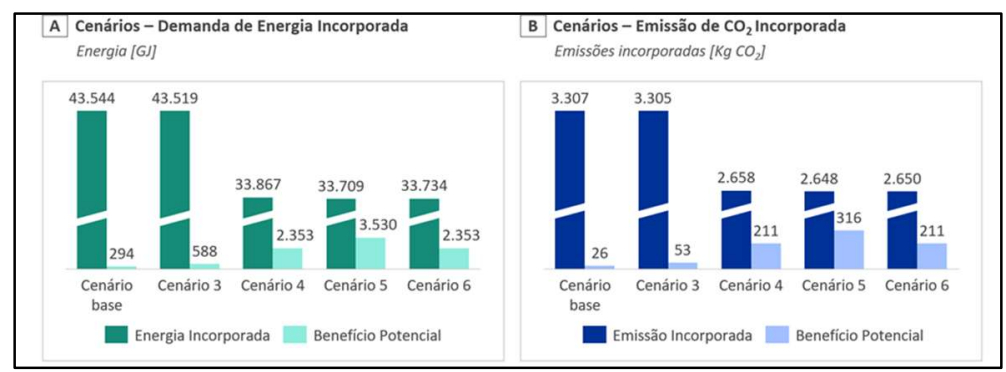

Figura 6: Energia e emissão incorporadas e benefícios em cada cenário.

No Cenário 3, percebe-se que praticamente não há uma redução no impacto ambiental gerado, tanto de demanda energética quanto de emissão de $\mathrm{CO}_{2}$, entretanto o seu benefício potencial é aproximadamente o dobro do Cenário 1 (cenário base). Ou seja, aumentar a taxa de reciclagem oferece um maior benefício para as futuras construções, entretanto não tem tanto reflexo na redução direta do impacto ambiental (demanda energética e emissão de $\mathrm{CO}_{2}$ ).

Além disso, é possível observar que os Cenários 4, 5 e 6 apresentam resultados muito similares para os impactos ambientais, com redução de aproximadamente $20 \%$ para a demanda energética e de aproximadamente $16 \%$ para emissão de $\mathrm{CO}_{2}$ quando comparados ao Cenário 1 (cenário base). Isso indica que aumentar a vida útil do PVC e das esquadrias, conforme simulado nos Cenários 4 a 6 , traz resultados muito positivos.

Com relação ao benefício potencial, percebe-se que ele é aproximadamente oito vezes maior do que 
aquele do cenário base, tanto para o Cenário 4 quanto para o Cenário 6. Já para o Cenário 5, o benefício potencial mostrou-se doze vezes maios do que o benefício do cenário base. Isso ocorre porque somente neste Cenário 5 foi considerado uma taxa de reciclagem de $60 \%$ (ou seja, $20 \%$ maior do que aquela adotada para o Cenário Base). Ou seja, fica evidente o benefício futuro que pode ser atingido ao alterar a taxa de reciclagem.

\section{CONSIDERAÇÕES FINAIS}

Através da análise dos diferentes cenários aplicados ao estudo de caso da escola, foi possível observar que um aumento de aproximadamente $20 \%$ na taxa de reciclagem dos resíduos de construção e demolição, somado a uma redução de $20 \%$ no percentual de perda na construção, traria grande benefício para o meio ambiente. Nessas condições, os impactos ambientais podem ser aproximadamente $20 \%$ menores do que o cenário base (cenário brasileiro) e com a possibilidade de trazer um benefício para as construções futuras de 12 vezes maior do que é alcançado atualmente (menos de 1\%). Assim, é possível constatar a importância de prezar pelo cuidado no transporte e manuseio de materiais na obra, a fim de garantir o mínimo possível de perdas, bem como assegurar que o material esteja em condições adequadas para ser reciclado.

Além disso, quando se trata especificamente do impacto ambiental em termos de demanda de energia e emissão de $\mathrm{CO}_{2}$ dos serviços executados ao longo da construção de uma edificação, a análise dos resultados do estudo de caso mostrou que as etapas de "Produção" e "Manutenção" são aquelas que possuem o maior peso, principalmente com relação à execução do sistema de vedação vertical interno e externo (paredes) e esquadrias. Assim, cabe ressaltar a importância de explorar medidas relacionadas a essas duas etapas e serviços para a redução do impacto ambiental no ciclo de vida de uma escola. Como mencionado anteriormente, algumas estratégias seriam utilizar esquadrias com maior vida útil, como as de madeira ao invés de esquadrias de alumínio, planejar uma ventilação adequada para evitar que a umidade do ar aumente a necessidade de manutenção da estrutura e substituir o concreto produzido in loco pelo préfabricado.

\section{REFERÊNCIAS}

ABIMCl. Associação Brasileira da Indústria de Madeira Processada Mecanicamente. Guia Especial de Portas da ABIMCI. 3 ed. Curitiba: ABIMCI, 2018.

ABNT. Associação Brasileira de Normas Técnicas. ABNT NBR ISO 14040 Gestão ambiental: Avaliação do ciclo de vida Princípios e estrutura. Rio de Janeiro: ABNT, 2009.

ABNT. Associação Brasileira de Normas Técnicas. ABNT NBR ISO 15575 Norma de Desempenho: Edificações Habitacionais. Rio de Janeiro: ABNT, 2013.

ADDIS, B.. Reuso de materiais e elementos de construção. São Paulo: Oficina de Textos, 2010.
ANGULO, S. C.. Caracterização de agregados de resíduos de construção e demolição reciclados e a influência de suas características no comportamento de concretos. São Paulo: Escola Politécnica da Universidade de São Paulo, 2005.

BOSSINK, B. A. G.; BROUWERS, H. J. H.. Construction waste: quantification and source evaluation. Journal of Construction Engineering and Management, Richmond, v.122, n.1, p.55-60, 1996. DOI: https://doi.org/10.1061/(ASCE)0733-9364(1996)122:1(55)

BRIBIÁN, I. Z.; CAPILLA, A. V.; USÓN, A. A.. Life cycle assessment of building materials: Comparative analysis of energy and environmental impacts and evaluation of the eco-efficiency improvement potential. Building and 
Environment, Zaragoza, v.46, n.5, p.1133-1140, 2011. DOI: https://doi.org/10.1016/i.buildenv.2010.12.002

BSI. British Standards Institution. Sustainability of construction works, assessment of environmental performance of buildings, calculation method. London: BSI, 2011.

BUTERA, S.; CHRISTENSEN, T. H.; ASTRUP, T. F.. Life cycle assessment of construction and demolition waste management. Waste Management, Lyngby, v.44, p.196-205, 2015. DOI: 10.1016/j.wasman.2015.07.011

CEARÁ. Secretaria municipal de Infraestrutura. Tabela de custos 026. Fortaleza: SEINFRA, 2019.

COLE, R. J.. Building environmental assessment methods: assessing construction practices. Construction Management and Economics, Vancouver, v.18, n.8, p.949-957, 2000. DOI: https://doi.org/10.1080/014461900446902

COLTRO, L.; MOURAD, A.; GARCIA, E. E. C.; QUEIROZ, G. C.; GATTI, J.; JAIME, S. B. M.. Avaliação do ciclo de vida como instrumento de gestão. Campinas, 2007.

HUBERMAN, N.; PEARLMUTTER, D.. A life-cycle energy analysis of building materials in the Negev desert. Energy and Buildings, Jerusalém, v.40, n.5, p.837-848, 2008. DOI: http://doi.org/10.1016/i.enbuild.2007.06.002

IPIRANGA, A. S. R.; GODOY, A. S.; BRUNSTEIN, J.. Introdução. RAM: Revista de Administração Mackenzie, São Paulo, v.12, n.3, p.13-20, 2011.
NG, W. Y.; CHAU, C. K.. New life of the building materialsrecycle, reuse and recovery. Energy Procedia, Hong Kong, v.75, p.2884-2891, 2015. DOI: https://doi.org/10.1016/j.egypro.2015.07.581

PINTO, T. P.. Metodologia para a gestão diferenciada de resíduos sólidos da construção urbana. Tese (Doutorado em Engenharia) - Escola Politécnica da USP, São Paulo, 1999.

PINTO, T. P.. Perdas de Materiais em Processos Construtivos Tradicionais. Dissertação (Mestrado em Engenharia Civil) - Universidade Federal de São Carlos, São Carlos, 1989.

POLITECNICO DI MILANO. Solid Waste Management and Treatment. DICA Environmental Section, Milano. 2018.

QUEIROZ, M. N.. Programação e controle de obras. Juiz de Fora, 2001.

SINAPI. Sistema Nacional de Pesquisa de Custos e Índices da Construção Civil. Cadernos técnicos do grupo. Brasília: Caixa Econômica Federal, 2019.

SOARES, S.; SOUZA, D.; PEREIRA, S.. A avaliação do ciclo de vida no contexto da construção civil. Coletânea Habitare, Porto Alegre, v.7, n.1985, p.96-127, 2006.

US EPA. United States Environmental Protection. Construction and demolition materials amounts. Building Related. Washington: US EPA, 2003.

A CBPC - Companhia Brasileira de Produção Científica (CNPJ: 11.221.422/0001-03) detém os direitos materiais desta publicação. Os direitos referem-se à publicação do trabalho em qualquer parte do mundo, incluindo os direitos às renovações, expansões e disseminações da contribuição, bem como outros direitos subsidiários. Todos os trabalhos publicados eletronicamente poderão posteriormente ser publicados em coletâneas impressas sob coordenação da Sustenere Publishing, da Companhia Brasileira de Produção Científica e seus parceiros autorizados. Os (as) autores (as) preservam os direitos autorais, mas não têm permissão para a publicação da contribuição em outro meio, impresso ou digital, em português ou em tradução. 